\title{
Why aren't more human resource leaders champions of diversity?
}

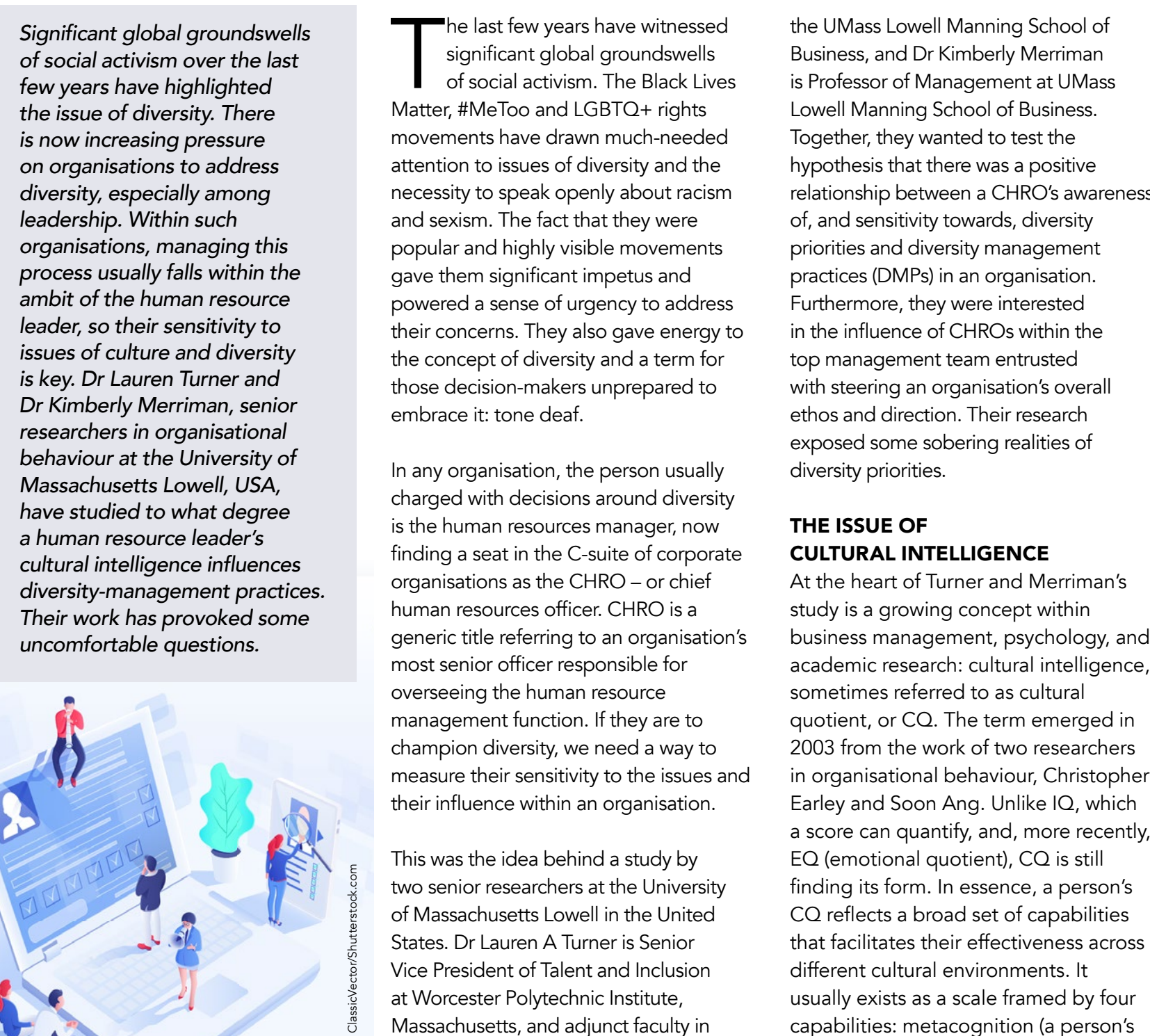

Significant global groundswells feccial activism over the last the issue of diversity There is now increasing pressure on organisations to address diversity, especially among leadership. Within such organisations, managing this process usually falls within the ambit of the human resource leader, so their sensitivity to issues of culture and diversity Dr Kimberly Merriman, senior researchers in organisational behaviour at the University of Massachusetts Lowell, USA, have studied to what degree a human resource leader's cultural intelligence influences diversity-management practices. their work has provoked so

he last few years have witnessed significant global groundswells
of social activism. The Black Lives Matter, \#MeToo and LGBTQ+ rights movements have drawn much-needed attention to issues of diversity and the necessity to speak openly about racism and sexism. The fact that they were popular and highly visible movements gave them significant impetus and powered a sense of urgency to address their concerns. They also gave energy to the concept of diversity and a term for those decision-makers unprepared to embrace it: tone de

In any organisation, the person usually is the human resources mound diversity finding a seat in the C-suite of corporat organisations as the $\mathrm{CHRO}$ - or chief human resources officer. CHRO is a generic title referring to an organisatio most senior officer responsible for overseeing the human resource management function. If they are to champion diversity, we need a way to measure their sensitivity to the issues a theri influence within an organisation.

This was the idea behind a study by two senior researchers at the University of Massachusetts Lowell in the United Vice President A Taner is Senior Wce President of Talont and Inclusion Massachusetts, and adjuct

the UMass Lowell Manning School of Business, and Dr Kimberly Merriman is Professor of Management at UMass Lowell Manning School of Business. Together, they wanted to test the hypothesis that there was a positive
relationship between a CHRO's awaren of, and sensitivity towards, diversity priorities and diversity management practices (DMPs) in an organisation. Furthermore, they were interested in the influence of $\mathrm{CHRO}$ s within the top management team entrusted with steering an organisation's overal ethos and direction. Their research exposed some sobeng realies of

THE ISSUE OF

CULTURAL INTELLIGENCE At the heart of Turner and Merriman's study is a growing concept within business management, psychology, and academic research: cultural intelligence, sometimes referred to as cultural quotient, or CQ. The term emerged in 2003 from the work of two researchers in organisational behaviour, Christopher Earley and Soon Ang. Unlike IQ, which a score can quantify, and, more recently, EQ (emotional quotient), $C Q$ is still finding its form. In essence, a person's $\mathrm{CQ}$ reflects a broad set of capabilities that facilitates their effectiveness across usually exists as a scale frants. It usually exists as a scale framed by four

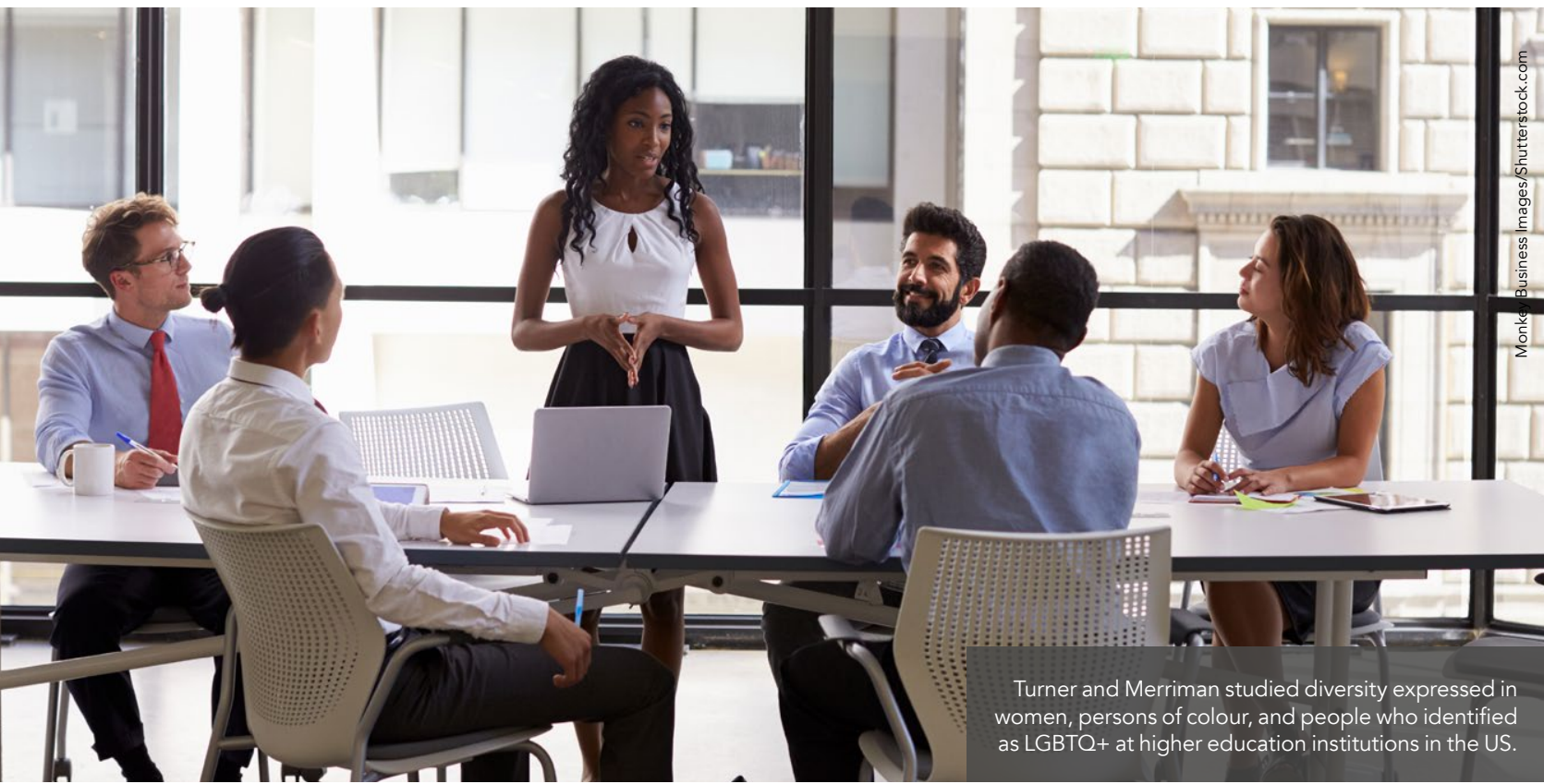

self-awareness of their assumptions cognitions (their knowledge and understanding of different cultures) motivation (their interest in experiencin other cultures), and behaviour (their

nuances of sub-cultures within nationally homogenous groupings, such as engineering and marketing teams within an organisation.

ability to apply that cultural knowled

What interested Turner and Merriman was diversity expr

their tenure, and how those $\mathrm{CHRO}$ inspired support from fellow members of the top management team when needed. Turner and Merriman's choice of higher education institutions makes sense: such institutions usually have a similar management structure to other

Much of the research into first centred around cultural piversity between

Diversity management practices go beyond legal requirements and therefore hold some degree of discretion for organisations. of colour, and people who identified as LGBTQ+ on the staff and faculty at higher of their study was tofold they whether the cultural intelligence of the CHROs at those institutions influenced diversity management practices during social identity groupings typ range from evidential differences between national cultures to

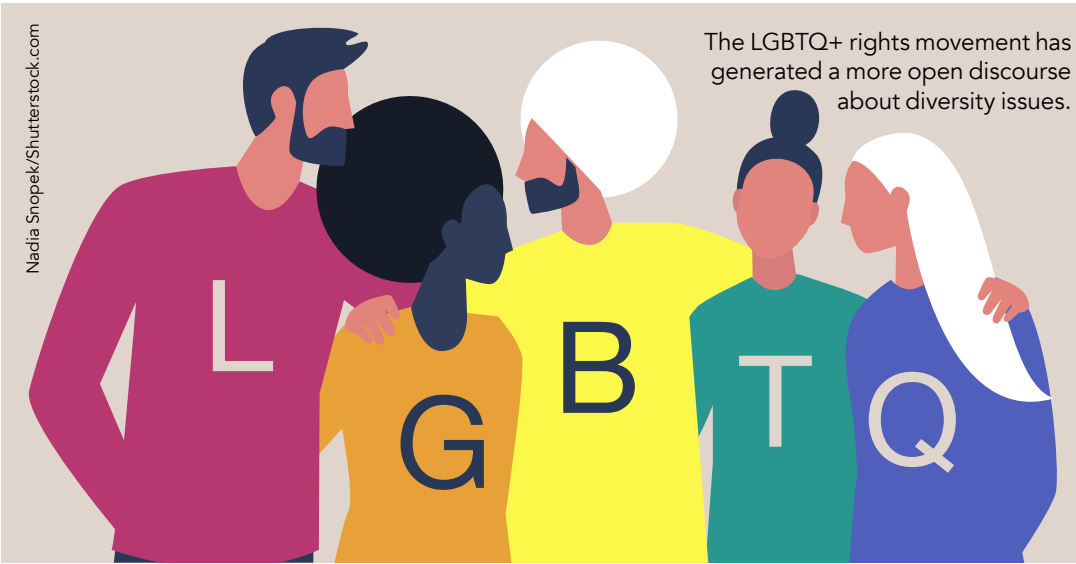

codes towards including boards and executive teams steering strategy. are supposedy enlightened mora diversity, and therefore should em

\section{TAPPING SENSITIVE DATA} Turner and Merriman sent an electronic colleges and universities across the US They ultimately attained 193 completed assessments of each CHRO's cultural intelligence and their use of certain transformative leadership behaviours presumably needed to 'sell' diversity management practices at the top. These responses were paired with public to caps and a second surveyed source to captre a ngorous account of each the

Multi-sourced information was also 


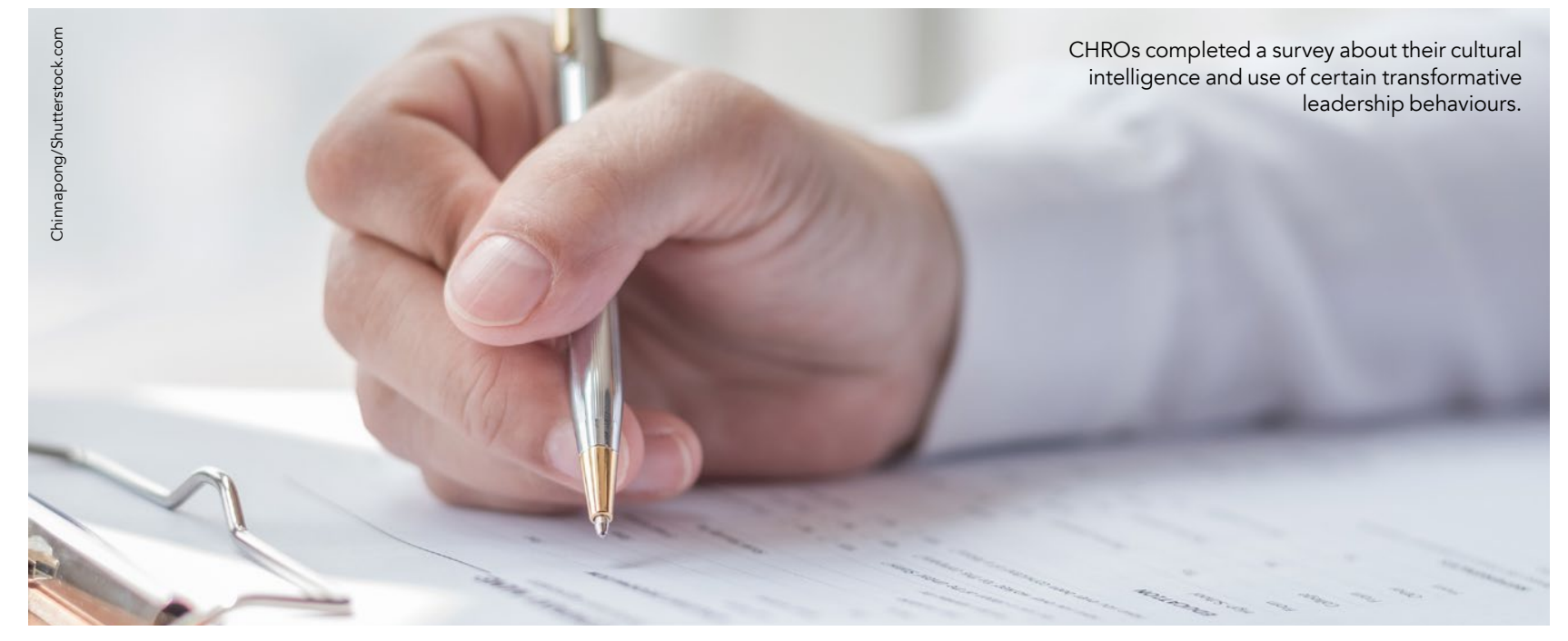

diversity management practices were established at each institution. These responsibility for the estabish of diversity manament, such as committees overseeng diversity initiatives; those that reduce bias in organisational decision-making, such as training programmes on diversity management for senior leaders; and practices that reduce social isolation such as mentoring and networking programmes. the sample organisations. CHRO differen iv practices within an industry already known for its attention to diversity.

The findings showed that influence mattered, that culturally intelligent CHROs outcomes through transformative persuasion of other senior leaders. - higher education - and as a crossand therefore unable to unearh clear lines of causality. However it does have broad implications: it underlines the importance of cultural intelligence in organisation's CHRO if critical diversity management practices are to take hold representative organisations. Therefore, it suggests cultural intelligence be translate their intentions into actu and produce socially healthier and more

It was evident that a CHRO's cultural $\begin{aligned} & \text { qualification } \\ & \text { for CHRO }\end{aligned}$

legal requirements

and therefore

of discretion for

organisations.

intelligence accounted for significant recruitment. It variance in the organisation's established the importance of cultural f these, the

diversity management practices. intelligence within Members of top management teams CHRO's tenure. often have distinct priorities the with diversity efforts. The same

WHAT MAKES AN EFFECTIVE CHRO? inherent abilities that underlie cultura

intelligence also seem aligned

Merriman's study confirmed both

of their predictions. Focusing on

the impact of the CHRO's cultural

intelligence by controlling for other

potential influences - such as diversity

management practices in place before

their tenure versus those created

during $i t$, their years in tenure and

therefore time to potentially engage

heir potential transformational

influence, and their annual operating

budgets - it was evident that a CHRO'

cultural intelligence accounted for

intelligence also seem aligned

with a particularly effective form of

transformative leadership behaviours

is needed. These behaviours include providing an inspirational vision to others, serving as an ideal role mode coaching instead of demanding, and figuratively meeting people

where they are at.

Like any research, Turner and

veriman's study is a small piece in a very big and complicated puzzle about

diversity management practices across management team beyond just the in order to supplant the need to 'sell' diversity practices at the top.

The study also prompts some able questions at a time when issues of diversity are still so sensitive yet critical to social discourse: why do almost three quarters of the CHROs lack formal membership on their institution's senior leadership team and why do only one quarter of the institutions provide training in diversity management for their board? And, if college and university campuses as bastions of diversity and human rights seem somewhat reluctant to elevate diversity management to the highest levels of leadership on their own campuses, what beyond their hallowed

\section{Behind the Research}

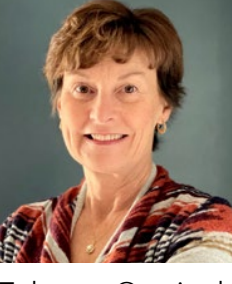

Dr Lauren A

Turner

|turner@wpiedu 0 @|turner UML

Wher-07786872

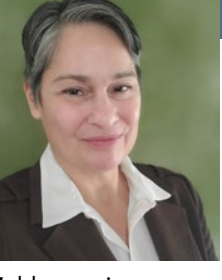

QkkMeriman
Dr Kimberly

Merriman

\section{Research Objectives}

The team's research shows the importance of cultural intelligence in effective human resource management (HRM)

Detail

Pr Lauren A Turner is Senior Vice President of Talent and Inclusion Mascester Polytechnic instiute, Tossachusets, and adchst faculy of Business. She has served in top eadership positions in HRM in higher education for over 30 years and was

\section{Personal Response}

\section{How are you planning to advance your research?}

II Our next study, currently underway, examines unique qualitative data from an organisational task force set up to address sexual harassment and equity during the 1980s. We are again dissecting how diversity practices becom established and will extrapolate implications for today. Surprisingly, many of the diversity issues from 30 years ago
still very much exist today.

Earley, PC, Ang, S, (2003) Cultural intelligence: Individu .

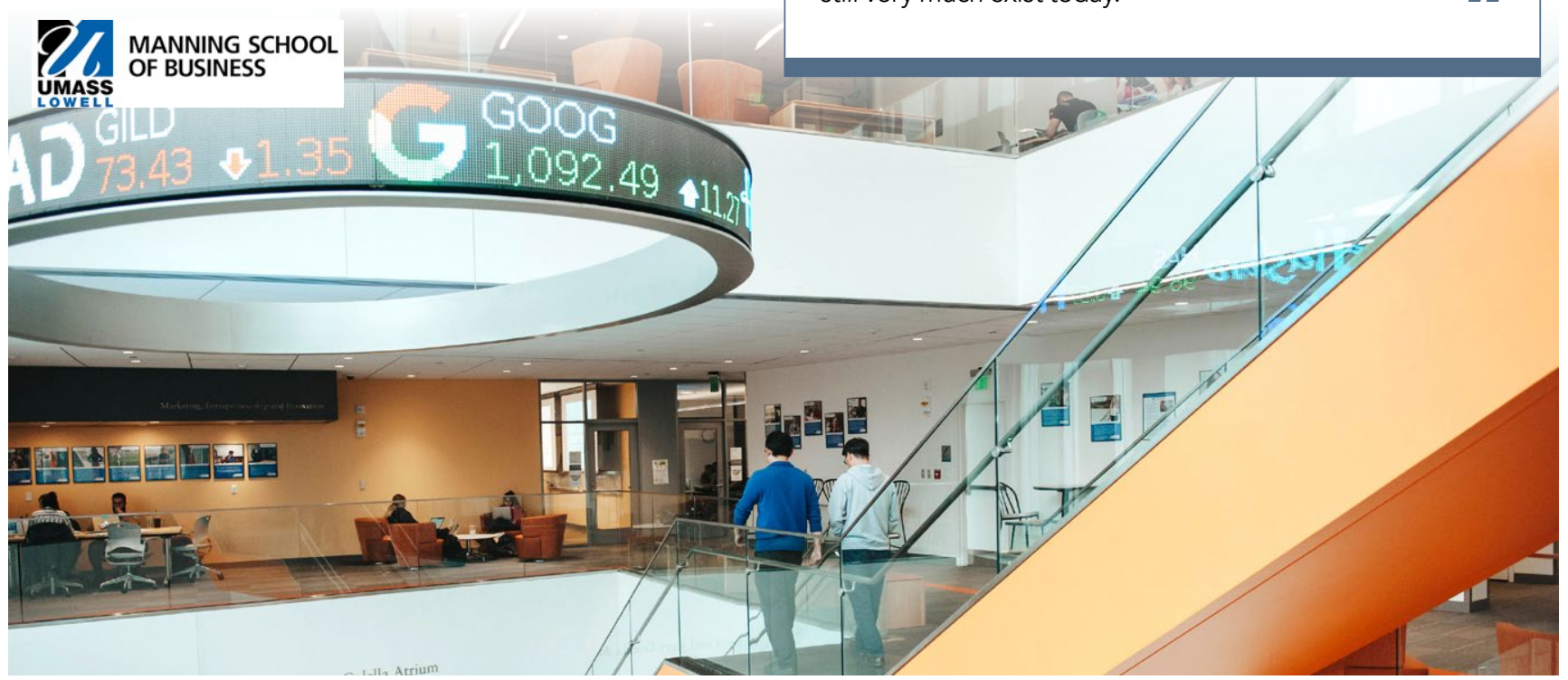

